\title{
Design and characterization of the MUSCAT detectors
}

\author{
V. Gómez-Rivera ${ }^{\mathrm{a}}$, P. A. R. Ade ${ }^{\mathrm{b}}$, P. S. Barry ${ }^{\mathrm{c}, \mathrm{d}}$, T. L. R. Brien ${ }^{\mathrm{b}}$, E. Castillo-Domínguez ${ }^{\mathrm{e}}$, D. \\ Ferrusca $^{a}$, P. Hargrave ${ }^{b}$, J. L. Hérnandez-Rebollar ${ }^{\mathrm{a}}$, A. Hornsby ${ }^{\mathrm{b}}$, D. Hughes ${ }^{\mathrm{a}}$, J. M. \\ Jáuregui-García ${ }^{\mathrm{a}}$, P. Mauskopfe, D. Murias ${ }^{\mathrm{a}}$, A. Papageorgiou ${ }^{\mathrm{b}}$, E. Pascale ${ }^{\mathrm{g}}$, A. Pérez ${ }^{\mathrm{a}}$, I. \\ Rodríguez $^{\mathrm{a}}$, S. Rowe ${ }^{\mathrm{b}}$, M. Tapia ${ }^{\mathrm{a}}$, C. Tucker ${ }^{\mathrm{a}}$, M. Velázquez ${ }^{\mathrm{a}}$, S. Ventura ${ }^{\mathrm{a}}$, and S. Doyle ${ }^{\mathrm{b}}$ \\ ${ }^{a}$ Instituto Nacional de Astrofísica, Óptica y Electrónica, Luis Enrique Erro 1, Santa María \\ Tonantzintla 72840 Puebla, Mexico \\ ${ }^{\mathrm{b}}$ School of Physics \& Astronomy, Cardiff University, The Parade CF24 3AA, Cardiff, United \\ Kingdom \\ ${ }^{\mathrm{c}}$ University of Chicago, 5640 S. Ellis Ave., Chicago IL, USA \\ dArgonne National Laboratory, 9700 S. Cass Ave., Lemont IL, USA \\ eSRON-Netherlands Institute for Space Research, Landleven 12, 9747 AD Groningen, \\ Netherlands \\ ${ }^{\mathrm{f}}$ Arizona State University, Tempe, Arizona, United States of America \\ ${ }^{g}$ Dipartimiento di Fisica, La Sapienza Universitá di Roma, Piazzale Aldo Moro 5, 00185 Roma, \\ Italy
}

\begin{abstract}
MUSCAT is a second-generation continuum camera for the Large Millimeter Telescope (LMT) "Alfonso Serrano", to observe at the $1.1 \mathrm{~mm}$ atmospheric window. The camera has 1500 background-limited, horn-coupled lumpedelement kinetic inductance detectors (LEKIDs) split across six arrays operating at 130-mK. The detector design for MUSCAT is based on a large-volume, double-meander geometry used as the inductive and two-polarization absorbing section of the LEKID resonator. In this paper we present the optical coupling of the meander to a choked waveguide output, the microwave design of the LEKID architecture, the device fabrication process and results demonstrating the detector sensitivity under a range of optical loads. Also presented is the performance of an aluminum absorbing layer used to minimize the optical cross-talk between detectors.
\end{abstract}

Keywords: Kinetic Inductance Detectors, Noise Equivalent Power, Superconductor Resonators

\section{INTRODUCTION}

Sub-mm / mm astronomy and non-invasive imaging applications have driven the maturity of Kinetic Inductance Detector (KIDs) technology. In terrestrial astronomy, NIKA2 was the first instrument based on detectors called Lumped Element Kinetic Inductance Detectors (LEKID's) [1]. LEKID's have demonstrated to be background limited detector, under different optical loads, typical for ground base observations $[2,3]$. LEKID's compared to superconductive bolometers (Transition Edge Sensors, TES) are easy to integrate in kilo-pixel arrays improving the overall Mapping Speed of the system by increasing the focal plane coverage. In applications such as passive imaging, advantage is taken that sub-mm / $\mathrm{mm}$ radiation is non-ionizing radiation and that certain materials become transparent in this frequency range. Therefore disciplines such as biomedical sensing, homeland security and non-destructive testing have taken advantage of the state of the art in sub-mm / $\mathrm{mm}$ astronomy. Such is the case of a video camera based on kinetic inductance detectors developed by the Cardiff University[4] that allows video at a rate of $2 \mathrm{~Hz}$, with 152 elements for a frequency of $350 \mathrm{GHz}$.

In this article, we present our development to design, fabricate and characterize an array of 1500 backgroundlimited pixels, which are to be installed in the second generation continuum camera MUSCAT [5] for the LMT

Further author information: (Send correspondence to V. Gómez-Rivera)

E-mail: vhgomez@inaoep.mx, Telephone: 2215712303 
to observe at 1.1 millimeter wavelength. In Sec. 2 we present the pixel design and optical coupling scheme. In Sec. 3 the fabrication process of 20 pixel detector array prototype is described. In Sec. 4 we describe the device characterization in order to show the noise equivalent power of the detector as well as the performance as a photon noise limited detector. Also the advantage of use an aluminum layer to decrease the optical cross-talk in neighbors detectors.

\section{DETECTOR DESIGN}

The MUSCAT detector design is based upon a horn coupled design where the horn output is coupled to a LEKID through an impedance matched choked structure as outline in Fig. 1. The LEKID's absorbing meander is designed such that efficient coupling is achieved to both polarizations and a quartz anti-reflection coating is used to impedance match the horn output to the silicon substrate. The optical band pass of this arrangement is set using a low pass metal mesh filter and the natural waveguide cutoff of the horn/waveguide feed [6]. This architecture allows the coupling of the optical power to a highly responsive inductive meander without the use of antennae or quasi-particles traps [1]. The MUSCAT LEKID has a large-volume, double-meander geometry that, together with the interdigitated capacitor (IDC), provides highly quality factors under optical loads of up to $100 \mathrm{pW}$. The simulations of the expected absorption spectrum have been carried out using ANSYS's HFSS software and the microwave feed-line coupling design was carried out using Sonnet software.

The detectors of the focal plane arrays will be distributed using a hexagonal-packing arrangement with a spacing of $1 \mathrm{f} \lambda$. Each detector forms a resonant microwave circuit with a $3 \mu \mathrm{m}$ wide inductive strip and an IDC capacitor, designed to resonate at a unique frequency. For large format array of resonators, the inductor is designed to be identical in every pixel while each IDC is tuned a unique capacitance by adjusting the length of the IDC fingers. The concept used, provides a simple and cost effective way of producing large format arrays with modest processing facilities. We tested the design into lab measurements with small prototypes devices consisting of a 20 pixels array each.

\subsection{Optical coupling design}

The model to obtain the absorption spectrum of the horn-coupled LEKID consists of a Choke-waveguide horn with cutoff frequency at $250 \mathrm{GHz}$ coupled to an anti-reflective quartz layer with an impedance of $Z_{\mathrm{AR}}=Z_{0} / \sqrt{\epsilon_{r}}=$ $194 \Omega$ and a silicon substrate with impedance of $Z_{\text {subs }}=110 \Omega$, where $Z_{0}$ is the vacuum impedance $(377 \Omega)$ and $\epsilon_{r}$ is the dielectric constant of substrate. The double-meander inductive absorber is fabricated from an aluminum film with sheet impedance of $1 \Omega / \square$ placed on the rear face of the silicon substrate, using the back illuminated configuration as shown in the Fig. 1. Between the anti-reflective and silicon substrates an aluminum metal layer is placed, this layer has an aperture concentric to the meander section and is used as a stray light absorber to minimise optical cross-talk between detectors.

The proposed arrangement maximises the use of the focal plane by focusing light onto the sensitive meander section of the LEKID avoiding illumination of non-sensitive elements such as the IDC and the readout feedline. The combination of horn coupling and the use of the stray light absorbing layer reduces the impact of off axis stray light that may propagate through the system. Two absorbing layers were tested. Both absorbing layers were fabricated from $40 \mathrm{~nm}$ Aluminium films with a sheet impedance of around $0.1 \Omega / \square$. The first was a solid sheet with a clear aperture etched behind each horn as shown in Fig. 1 schematic and HFSS model. The second comprised etching a meander structure into the solid sheet to increase the effective sheet impedance to better match the silicon substrate.

\subsection{Transmission line coupling design}

The resonant microwave circuit is capacitively-coupled to a coplanar waveguide (CPW) $50 \Omega$ feedline using capacitive side couplers. The resonator is fabricated from an aluminum film with sheet impedance of $1 \Omega / \square$ and $T_{\mathrm{c}}=1.4 \mathrm{~K}$ and kinetic inductance of $L_{\mathrm{s}} 0.9 \mathrm{pH} / \square$ at $100 \mathrm{mK}$ bath temperature. We used a compact resonator design to achieve $1 f \lambda$ pixel spacing for the optical design ( $3 \mathrm{~mm}$ pixel-to-pixel center spacing for 1.1 $\mathrm{mm}$ wavelength) as is showed in Fig. 2. In this design we used two IDC's configurations to cover the readout bandwidth and by adjusting the side couplers length we keep the coupling quality factor at $\sim 50000$ with resonance frequency $\left(f_{0}\right)$ spacing within the range of $\sim 1.4$ to $\sim 2.4 \mathrm{MHz}$, matching the MUSCAT readout band $\sim 0.5-1.1 \mathrm{GHz}$. 

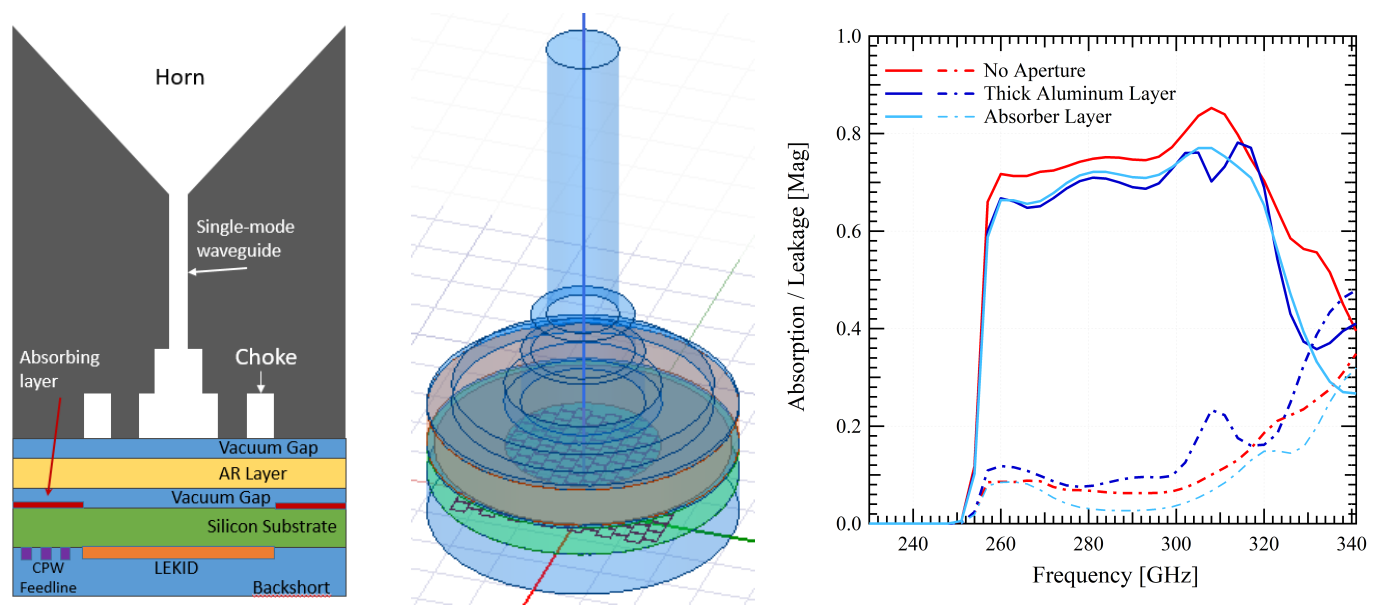

Figure 1. Left. Cross-sectional configuration of a single MUSCAT pixel on the focal plane, the vacuum gaps shown in this schematic are included to illustrate small imperfections $(50 \mu \mathrm{m})$ in the assembly of the detector package due to wafer bowing. These effects are included in the HFSS simulation model. Center. HFSS simulation model of single horn-coupled LEKID. Right. Absorption and leakage spectrum for a single coupled LEKID. Continuous lines are the absorption spectrum and dashed-dotted lines are the potential leakage of light that can reach another LEKID. This plot represent three different scenarios, first (red lines) is without the use of any aperture, second (blue lines) is using an aperture over a thick aluminum layer and third (cyan) is using an absorber aluminum layer.
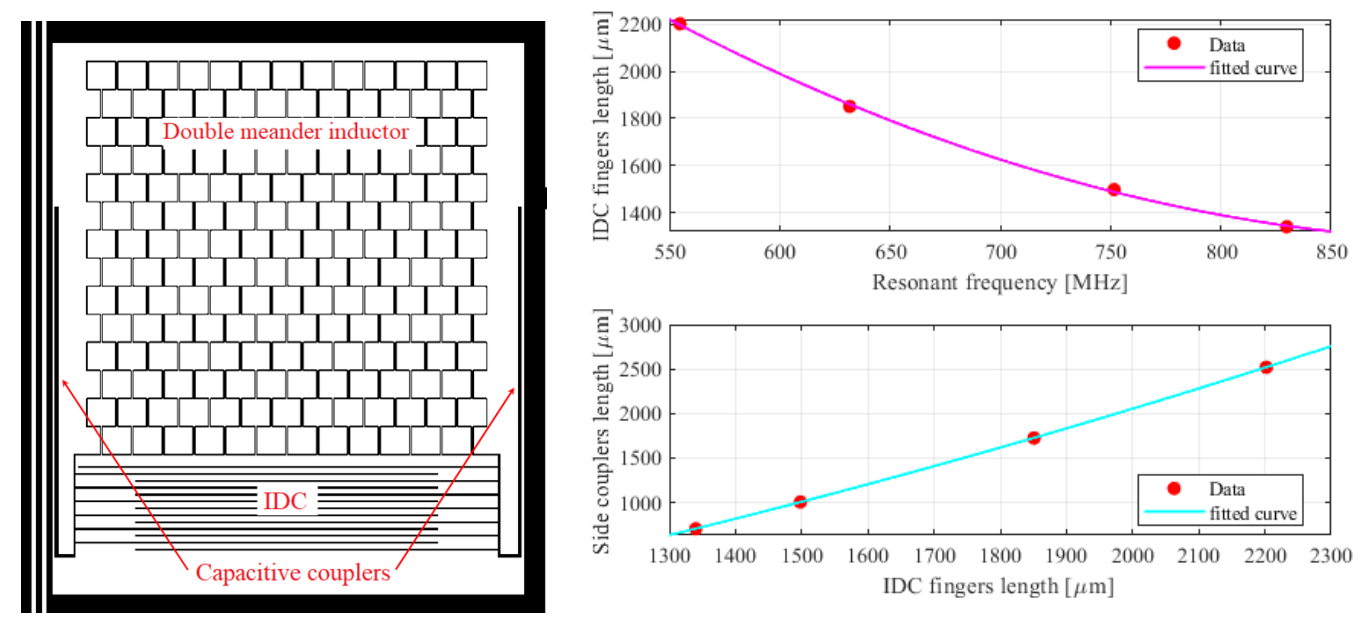

Figure 2. Left. Schematic of the MUSCAT detector capactively coupled to readout feedline using the side couplers. Right. Data of four resonators simulated with $Q_{\mathrm{r}}$ fitted to 50000 for the low frequencies band and the trendlines used to predict the length for the side couplers and the capacitor fingers given a resonant frequency. In this way we can control the $Q_{\mathrm{r}}$ around to 50000 .

\section{FABRICATION PROCESS}

We designed a prototype 20-pixel array in order to measure the performance of the LEKIDs as shown in the Fig. 3. Each array spanning $\sim 500 \mathrm{MHz}$ of readout bandwidth, the resonance frequencies are grouped in two bands by adjusting the configuration of the IDC. 12 resonators are located in a low-frequency band between $\sim 556$ and $\sim 773 \mathrm{MHz}$ with $f_{0}$ spacing of $\sim 18 \mathrm{MHz}$; and 8 resonators in a high-frequency band between $\sim 840$ and $\sim 1100 \mathrm{MHz}$ and $f_{0}$ spacing of $\sim 23 \mathrm{MHz}$. The fabrication process was carried out by the University of Chicago using standard micro-fabrication techniques. The LEKIDs are patterned from a $25 \mathrm{~nm}$ thick aluminum film and $3 \mu \mathrm{m}$ width traces. The aluminium was deposited on a $100 \mathrm{~mm}$ diameter, high-resistivity, silicon substrate $\left(\rho \sim 2 \cdot 10^{4} \Omega \cdot \mathrm{cm}\right)$ with $300 \mu \mathrm{m}$ thickness, designed to maximise optical coupling in combination with 
a backshort structure. A RCA clean was implemented over the silicon wafer, prior to the aluminum deposition, a pre-deposition of $30 \mathrm{~nm}$ of titanium was made with the wafer unexposed to reactively pump chamber. The aluminum film is deposited with a chamber pressure of $1 \cdot 10^{-7}$ Torr. The array was patterned using direct write lithography for both, the front and backsides of the array. Bridge structures were added across the CPW feed line to prevent slot-line mode propagating. These were fabricated using a liftoff techniques to deposit $\mathrm{SiO} 2$ dielectric pads spanning the feedline. Aluminium bridges are then deposited over the $\mathrm{SiO} 2$ pads again using a liftoff technique.

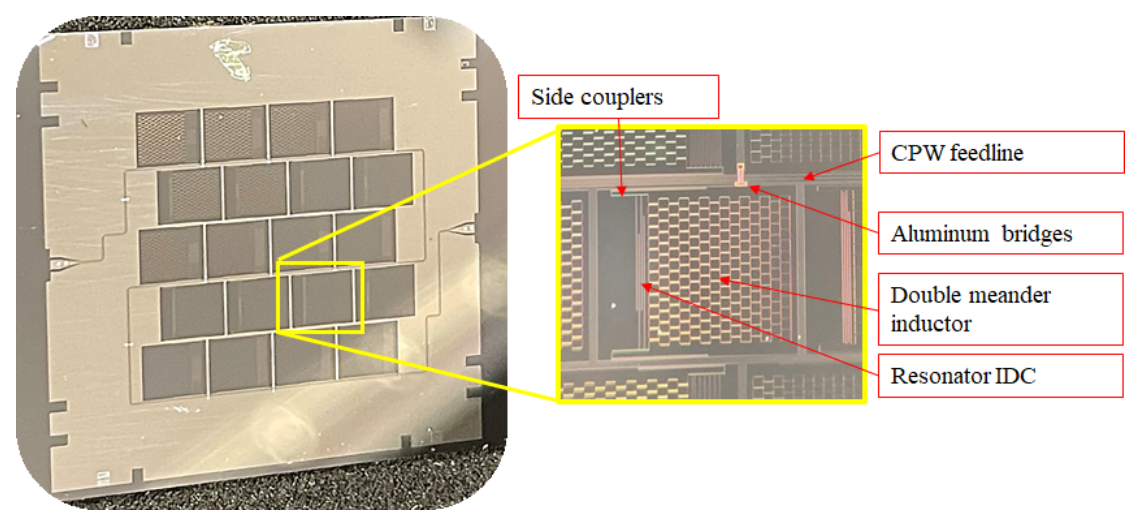

Figure 3. Left. Photo of a $20 \mathrm{~mm}$ x $20 \mathrm{~mm}$ prototype array with 20 pixels after of the fabrication process. Right. Expanded view that shows the layout of a single MUSCAT pixel.

\section{LOW TEMPERATURE CHARACTERIZATION}

For the low temperature measurements, the small array was cooled using a miniature dilution fridge to $130 \mathrm{mK}$, this temperature was selected to match the operating temperature of the MUSCAT instrument [7]. To couple the radiation from a black body, a horn block was made with 20 choke-waveguide feed-horn apertures of which two were subsequently blanked by a copper plate. Thus, optical radiation was coupled into 18 resonators leaving 2 resonators blind detectors to measure the stray light in the array. The assembled structure is shown in the Fig. 4. This horn block is a 20-horn version of the final MUSCAT horn block design.
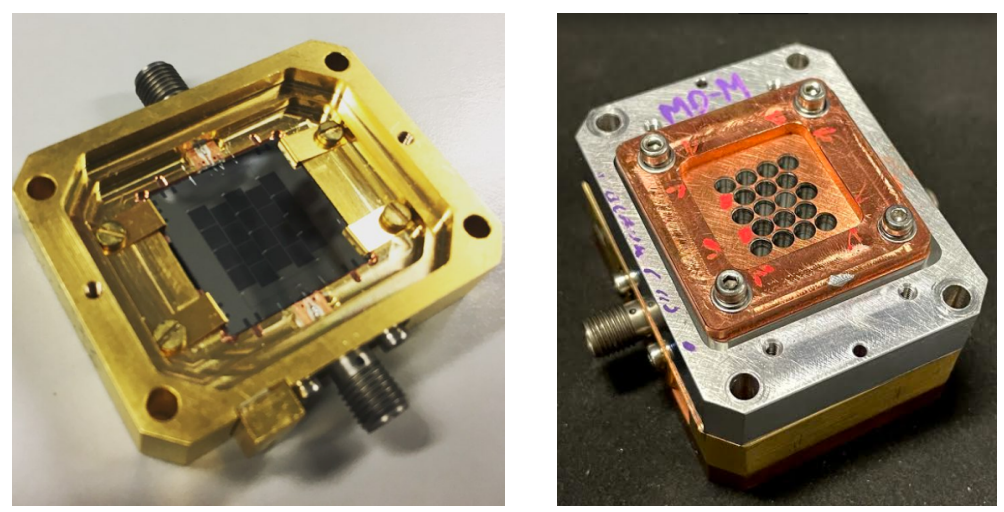

Figure 4. Left. $20 \mathrm{~mm}$ x $20 \mathrm{~mm}$ prototype array mounted in a detector holder, viewed from the back-short (removed) side. Right. Top-view of the manufactured feed-horn array for the lab measurements with 18 apertures, a copper plate was added to block two of the feedhorns. The red dots are the position of the 2 blinded detectors.

\subsection{Resonance frequencies and coupling quality factors}

To identify the resonant frequency of each resonator, resonances were first located via a frequency sweep of the $S_{21}$ scattering parameter using a vector network analyzer (VNA), followed by a sweep around each resonance 
using a homodyne system, from which we obtain the time streams of I and Q signals. With these signals we can calculate the magnitude of the $S_{21}$ transmission parameter $\left(S_{21}=\sqrt{I^{2}+Q^{2}}\right)$ to estimate quality factors of the resonator. When measuring timestreams, the homodyne system has a high sampling frequency that allows measurements of quasi-particles lifetime and ringtone time of the resonator [8], these parameters together with measurements of the resonance frequency fluctuations [9] allow the noise characterization of the resonator to be measured. Fig. 5 shows the resonances frequencies of each detector in the array as well as the resonator quality factor $\left(Q_{\mathrm{r}}\right)$.
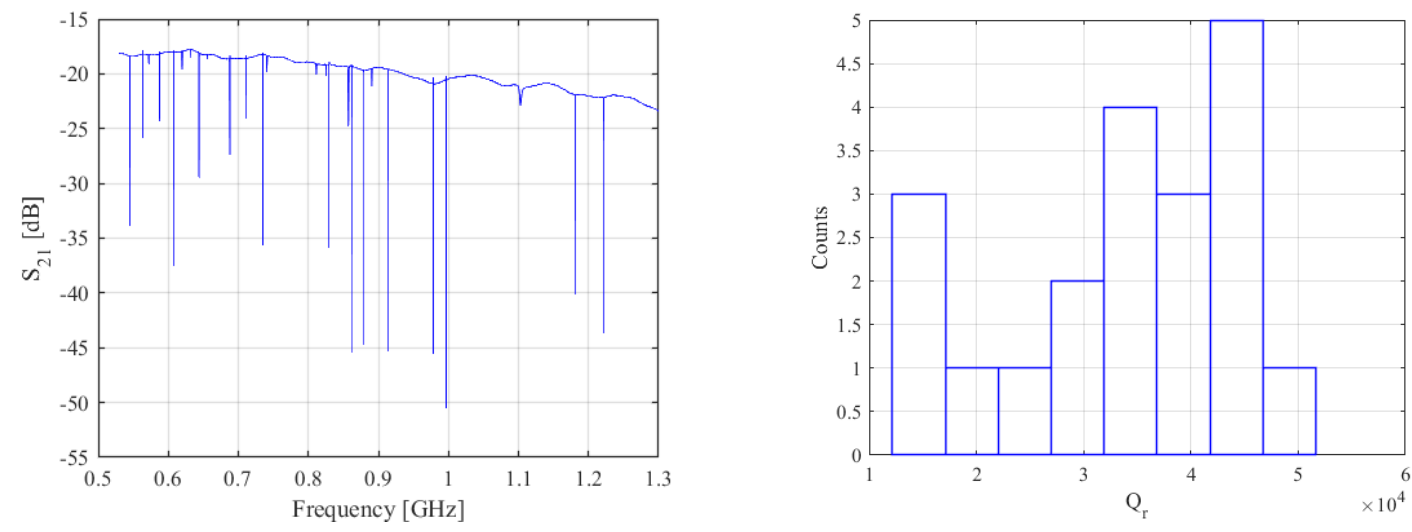

Figure 5. Left. VNA frequency sweep of the $S_{21}$ parameter of the MUSCAT prototype small array with 20 resonances detected. Right. Resonator quality factors of the 20 resonances detected, discarding the low $Q_{\mathrm{r}}$, the average is $\sim 37000$.

In this array we detected 20 resonances, 17 with good resonance depth and 3 with shallower resonance, also we see others small false resonances probably due to cross-talk effects, the resonator quality factor is $\sim 37000$ on average discarding the low $Q_{\mathrm{r}}$ of 3 resonators with shallower resonance.

\subsection{Noise Equivalent Power measurements}

For the measurements of responsivity and optical noise equivalent power (NEP), the detectors were radiated with a blackbody calibration source with temperatures $\left(T_{\mathrm{BB}}\right)$ from $\sim 7$ to $\sim 70 \mathrm{~K}$. We calculated the power spectral density (PSD) of the resonance frequency fluctuation $\left(d f_{0}\right)[9,8]$ at different values of $T_{\mathrm{BB}}$. From the PSD we can analyse the typical frequency noise of each detector, i.e. the two-Level system noise $\left(N_{\mathrm{TLS}}\right)$, generationrecombination noise $\left(N_{\mathrm{gr}}\right)$ and the amplifier noise $\left(N_{\mathrm{amp}}\right)$. Parameters such as the quasi-particles lifetime $\left(\tau_{\mathrm{qp}}\right)$ and the resonator ringtone time $\left(\tau_{\text {res }}\right)$ are also found. To estimate the NEP, it is necessary to measure the detector responsivity under illumination of a known optical power, i.e. a black body source. We calculated the NEP using the frequency noise spectral density divided by the detector responsivity $d f_{0} / d P$ and normalizing it with the time constants $\tau_{\text {qp }}$ and $\tau_{\text {res }}$ according to [10,8], Fig. 6 shows the PSD and the NEP as a function of the black body temperature.

Under good observing conditions the typical detector load is of order $9 \mathrm{pW}[11,12,13]$ equivalent to an effective sky temperature of order 20K. As shown in Fig. 7, this device shows background limited performance across this sky temperature range and hence is optimised for use at the LMT site.

We compare the detector performance with the expected photon noise including both shot- and wave-noise terms, and regeneration noise at different $T_{\mathrm{BB}}[14]$ as well as the contribution of the amplifier noise (Fig. 7 left), demonstrating photon noise limited performance of the horn-coupled LEKID. We measure the NEP as a function of $T_{\mathrm{BB}}$ averaging between $0.4-3 \mathrm{kHz}$ to avoid the $1 / f$ noise due to the readout channel.

To estimate the leaked power received from the blinded pixels we need to consider the variation of the LEKID response with absorbed optical power. The quasiparticle lifetime scales with optical power absorbed as $\tau_{\mathrm{qp}} \propto \sqrt{P_{\mathrm{opt}}}[15]$. This dependence leads to a nonlinear response of the LEKID as the quasiparticles generated through absorbed optical power is dependent on $\tau_{\mathrm{qp}}$. 

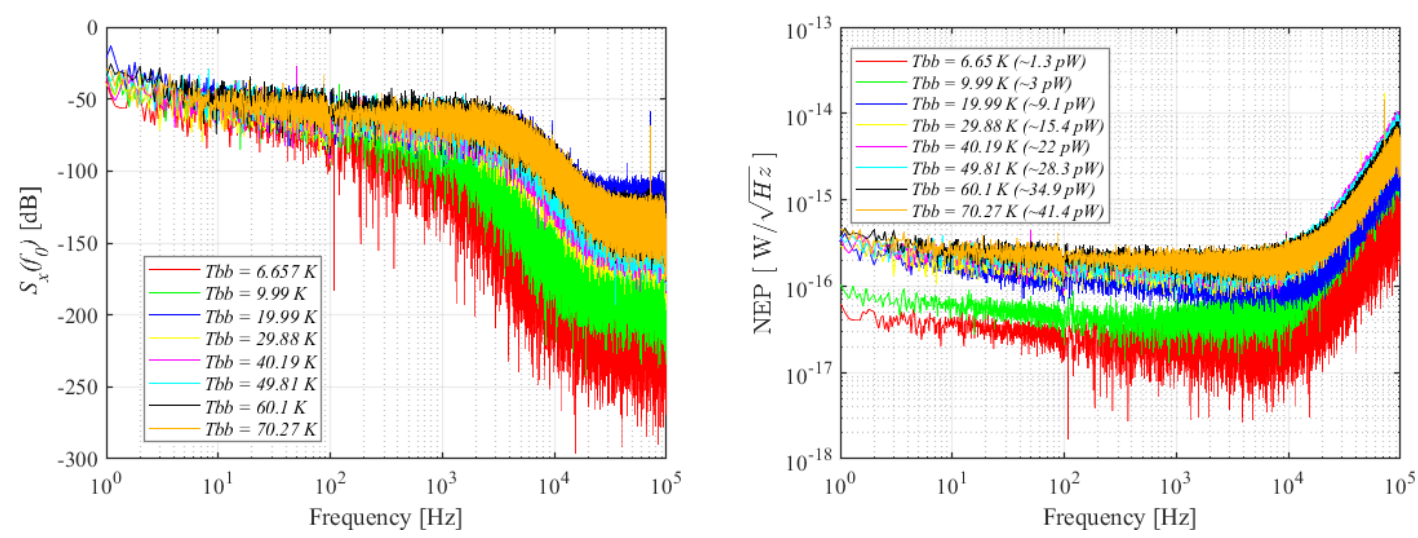

Figure 6. Measured power spectral density (left) and noise equivalent power (right) at different black body temperatures. Both plots correspond at the same detector at bath temperature of $130 \mathrm{mK}$, the NEP measurement at $T_{\mathrm{BB}}=6.65 \mathrm{~K}$ corresponding to an optical power incident upon the absorber of $\sim 1.37 \mathrm{pW}$ is $\sim 4 \cdot 10^{-17} \mathrm{~W} / \sqrt{\mathrm{Hz}}$ averaging between 0.4 - $3 \mathrm{kHz}$. From the PSD, we calculated a $\tau_{\mathrm{qp}} \sim 200 \mu \mathrm{s}$ and generation-recombination noise level $\sim 2.7 \cdot 10^{-18} \mathrm{~W} / \sqrt{\mathrm{Hz}}$.

$$
N Q P_{o p t}=\frac{\eta P_{a b s} \tau_{q p}}{\Delta}
$$

Where $P_{\text {abs }}$ is the optical power absorbed in the LEKID meander, $\eta$ is a pair breaking efficiency factor and $\Delta$ is the Copper pair binding energy. The plot in Fig. 7, compares the average response of the blind pixels and the illuminated pixels to the blackbody temperature for the array where the solid aluminium aperture was used and the array where an absorbing structure was used. Here we plot the response in terms of fractional frequency shift (FFS).

$$
F F S=\frac{F_{0}^{\prime}-F_{0}}{F_{0}}
$$

Where $F_{0}$ is the resonant frequency at the lowest blackbody temperature and $F_{0}^{\prime}$ is the measure resonant frequency response to changing blackbody temperature. Using FFS removes any dependence on variation in response with device resonate frequency. We then fit a model where we assume the blind pixels absorb some fraction of the power incident on an illuminated pixel:

$$
P_{\text {Blind }}=P_{\text {illuminated }} X T,
$$

Where $X T$ is the cross-talk leakage that varies between 0 and 1 . Using this model we find that in the array where solid apertures are used fabricated form an aluminium film of sheet impedance $\approx 0.1 \Omega / \square$ we see a leakage power of approximately $14 \%$ of the power incident on a single illuminated pixel. This is reduced to approximately $2 \%$ in the case where the absorbing structure with higher effective sheet impedance is used.

The photon and generation-recombination noise dominate in the range of incident power from $\geq 100$ fW to $20 \mathrm{pW}$, as we can see in the Fig. 7 left, we find numerically calculated an optical efficiency of the detector of $\eta_{\text {det }}=0.8 \pm 0.2$ in agreement with the $3 \mathrm{D}$ electromagnetic simulations. In the sub- $\mathrm{mm} / \mathrm{mm}$ region, the sky and optical loading power for a ground-based telescopes, such as the LMT, is in the order of 1 to $20 \mathrm{pW}$ [14], therefore operating at the LMT, the horn-coupled LEKID will be limited by this noise source. Also, we show (Fig. 7 right) the effect of the aluminum absorber used to decrease the stray light in the array and, therefore decrease the optical crosstalk. The FFS calculated from the responsivity measurements with the black body source, shows that the reduction of the stray light is in both, illuminated and blinded detectors with a ratio of responsivity blinded/illuminated of $2 \%$ using the absorbing layer and $14 \%$ without this. 

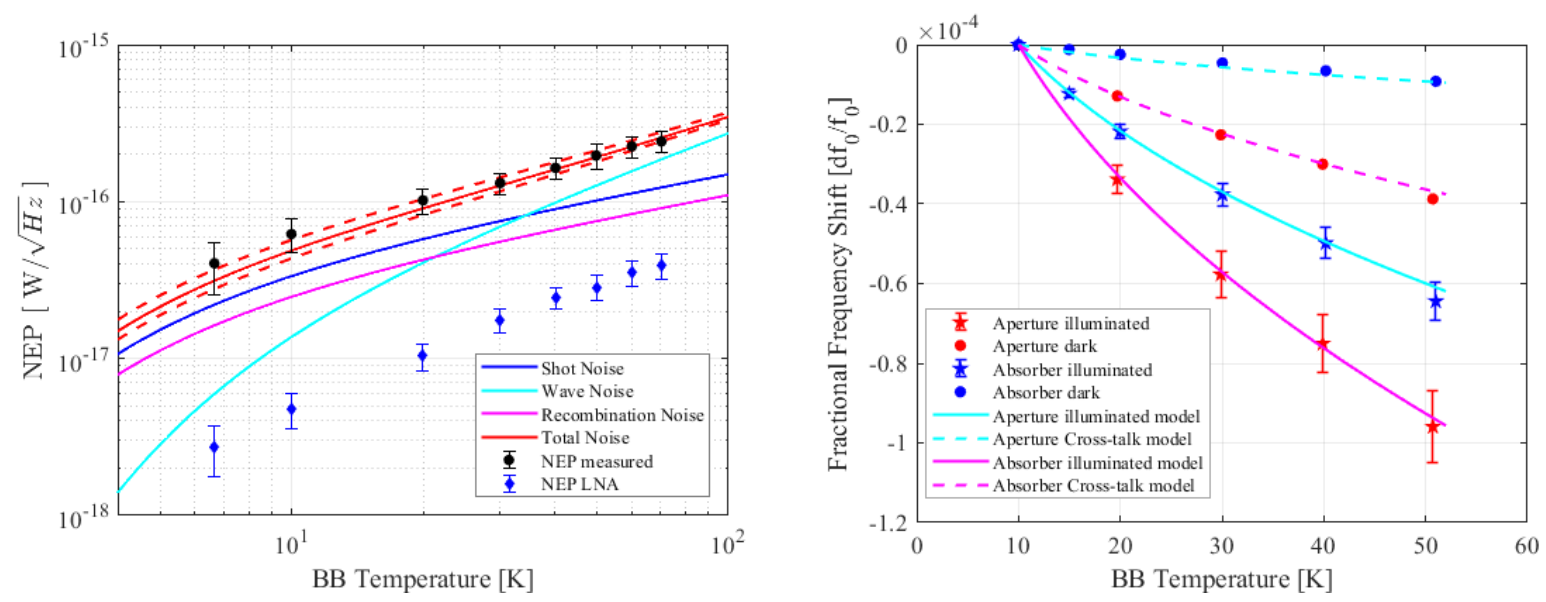

Figure 7. Left. NEP average $(0.4-3 \mathrm{kHz})$ as a function of $T_{\mathrm{BB}}$, the black and blue dots are the NEP measured of the detector and the low noise amplifier respectively. Continuos lines are the sources that contribute to total noise (red line) as well as the amplifier noise. We find a detector optical efficiency of $\eta_{\text {det }}=0.8$ with \pm 0.2 limits (red dashed lines). Right. Fractional frequency shift at base temperature of $T_{\mathrm{BB}}=10 \mathrm{~K}$ for all detectors in the array, grouping the illuminated detectors (mean, stars with error bars) and the blind detector. The plot compare the FFS between the array with clear apertures using an aluminum solid layer and the other using the aluminum absorber.

\section{CONCLUSIONS}

We have showed the design and characterization at $130 \mathrm{mK}$ of the MUSCAT detectors and also we showed the detector performance as a function of the black body power in the range of $\sim 1$ to $\sim 40 \mathrm{pW}$ where we see that the detector performance will be limited by photon noise when operating at the LMT. We have calculated a high optical efficiency $\eta_{\text {det }}=0.8 \pm 0.2$ for this horn-coupled LEKID system fabricated from Aluminum films on bulk Silicon substrates. This has provided a simple detector architecture for fabricating large format arrays for the next generation of cameras for the LMT based upon LEKID technology. Finally, we showed the advantage of use an absorber layer to decrease the stray light in the array and prevent the optical cross-talk, improving the false detection between neighbors detectors.

\section{ACKNOWLEDGMENTS}

We acknowledge RCUK and CONACYT through the Newton Fund (grant no. ST/P002803/1), CONACYT for support the fellowship for the instrument scientist (grant no. 053), Chase Research Cryogenics for the development of the sub Kelvin coolers and to XILINX Inc. for the donation of the FPGAs used for the ROACH2 boards.

\section{REFERENCES}

[1] S. Doyle, P. Mauskopf, J. Zhang, A. Monfardini, L. Swenson, J. J. A. Baselmans, S. J. C. Yates, and M. Roesch, "A review of the lumped element kinetic inductance detector",Proc.SPIE 7741, p. 10, 2010.

[2] Adam, R., Adane, A., Ade, P. A. R., et al."The NIKA2 large-field-of-view millimetre continuum camera for the 30 m IRAM telescope", 2018, A\&A, 609, A115. doi:10.1051/0004-6361/201731503

[3] Perotto, L., Ponthieu, N., Macías-Pérez, J. F., et al. "Calibration and performance of the NIKA2 camera at the IRAM 30-m telescope" 2020, A\&A, 637, A71. doi:10.1051/0004-6361/201936220

[4] Rowe,Sam and Pascale,Enzo and Doyle,Simon and Dunscombe,Chris and Hargrave,Peter and Papageorgio,Andreas and Wood,Ken and Ade,Peter A. R. and Barry,Peter and Bideaud,Aurélien and Brien,Tom and Dodd,Chris and Grainger,William and House,Julian and Mauskopf,Philip and Moseley,Paul and Spencer,Locke and Sudiwala,Rashmi and Tucker,Carole and Walker,Ian, "A passive terahertz video camera based on lumped element kinetic inductance detectors",Review of Scientific Instruments, vol. 87, pp. 033105, 2016. 
[5] E. Castillo-Dominguez, P. Ade, P. S. Barry, T. Brien, S. Doyle, D. Ferrusca, V. Gomez-Rivera, P. Hargrave, A. Hornsby, D. Hughes, Philip Mauskopf, P. Moseley, E. Pascale, A. Perez-Fajardo, G. Pisano, S. Rowe, C. Tucker, M. Velazquez. "Mexico-UK Sub-millimeter Camera for Astronomy", Journal of Low Temperature Detectors, vol. 193, pp. 1010-1015, 2018.

[6] Thomas L. R. Brien, Peter A. R. Ade, Peter S. Barry, Edgar Castillo-Domìnguez, Daniel Ferrusca, Thomas Gascard, Victor Gómez, Peter C. Hargrave, Amber L. Hornsby, David Hughes, Enzo Pascale, Josie D. A. Parrianen, Abel Perez, Sam Rowe, Carole Tucker, Salvador Ventura González, and Simon M. Doyle, "MUSCAT: the Mexico-UK Sub-Millimetre Camera for AsTronomy", Proc. SPIE 10708, 2018.

[7] T. L. R Brien et al .,"Pre-deployment Verification and Predicted Mapping Speed of MUSCAT", Millimeter, Submillimeter, and Far-Infrared Detectors and Instrumentation for Astronomy X, SPIE (these proceedings), 2020

[8] Marcial Becerril, "Readout and characterization system for kinetic inductance detectors (KID) of the MUSCAT project",Master thesis, INAOE, 2019.

[9] Sam Rowe, "Passive terahertz imaging with lumped element kinetic inductance detectors",PhD thesis, Cardiff University, 2015.

[10] P.J. de Visser, J.J.A. Baselmans, P. Diener, S.J.C. Yates, A. Endo, and T.M. Klapwijk, "Generationrecombination noise: The fundamental sensitivity limit for kinetic inductance detectors",Journal of Low Temperature Physics vol. 167, p. 335-340, 2012.

[11] T. A. Perera, T. C. Chen, E. S. Cheng, D. A. Cottingham, T. M. Crawford, T. P. Downes, F. M. Finkbeiner, D. J. Fixsen, D. W. Logan, S. S. Meyer, E. H. Sharp, R. F. Silverberg, G. W. Wilson, "Optical performance of frequency-selective bolometers",Applied Optics, Applied Optics 45,p. 7643-7651, 2006.

[12] G. W. Wilson, J. E. Austermann, T. A. Perera, K. S. Scott, P. A. R. Ade, J. J. Bock, J. Glenn, S. R. Golwala, S. Kim, Y. Kang, D. Lydon, P. D. Mauskopf, C. R. Predmore, C. M. Roberts, K. Souccar, M. S. Yun, "The AzTEC mm-wavelength camera",MNRAS Vol. 376, p. 807-818, 2008.

[13] G. W. Wilson, "The Spectral Energy Distribution Camera for the LMT",SPIE, Vol 4855, p. 583-593, 2003.

[14] S. J. C. Yates, J. J. A. Baselmans, A. Endo, R. M. J. Janssen, L. Ferrari, P. Diener, A. M. Baryshev,"Photon noise limited radiation detection with lens-antenna coupled microwave kinetic inductance detectors",Applied Physics Letter, Vol 99, 2011

[15] P.J. de Visser, J.J.A. Baselmans, J. Bueno, N. Llombart, T.M. Klapwijk,"Fluctuations in the electron system of a superconductor exposed to a photon flux" Nature Communications 5, 3130, 2013. 